\section{Caracterização da oferta de serviços especializados em hospitais de referência regional: uma contribuição para a regionalização da assistência à saúde no SUS}

\author{
A characterization of the specialized \\ services on offer in regional referral \\ hospitals: a contribution to the \\ regionalization of Brazilian public health \\ care
}

Luciana Santos Dubeux 1

Eduardo Freese de Carvalho 2
1,2 Centro de Pesquisas Aggeu Magalhães. Fundação Oswaldo Cruz. Av. Prof. Morais do Rego, s.n. Cidade Universitária. Recife, PE, Brasil. CEP: 52.041-060. E-mail: 1dubeux@cpqam.fiocruz.br

\begin{abstract}
Objectives: to characterize the specialized services offered in regional hospitals according to the type of care provided and their limitations.

Methods: a normative evaluation was carried out in ten regional hospitals in Pernambuco, by way of interviews with directors and doctors. Questions dealt with emergency/urgent care, care for women with high-risk pregnancies, the provision of complementary tests and elective surgery, and the types of professionals and hospital beds available.

Results: it was observed that the hospital care offered is not in conformity with the aims of the State Health Care Regionalization Model. Emergency/ urgent care mostly takes the form of clinical cases or low-complexity surgery, such as treatment for light wounds or appendectomies. Health care for pregnant women is restricted to deliveries without complications in most hospitals and there is a need to take on new staff (such as cardiologists and neurologists) in order to ensure that adequate care is provided. With regard to hospital beds, the highest ratio was 0.6 per 1.000 inhabitants at the Salgueiro Regional Hospital, with intensive care being concentrated in only three units.

Conclusions: limitations with regard to infrastructure and work processes impair the provision of services and the functioning of regional hospitals and consequently have an impact on the care provided at the States regional health units.
\end{abstract}

Key words Health evaluation, Regional health planning, Hospital care

\section{Resumo}

Objetivos: caracterizar a oferta de serviços especializados em hospitais regionais, considerando os tipos de assistência prestada e os fatores limitantes.

Métodos: avaliação normativa nos dez hospitais regionais de Pernambuco, mediante entrevistas com diretores e médicos. Foram abordadas questões referentes à assistência nas urgências/emergências, à atenção à gestante de alto risco, à oferta de exames complementares e de cirurgias eletivas, aos profissionais e aos tipos de leitos hospitalares.

Resultados: observou-se que a assistência hospitalar ofertada não está conforme o preconizado pelo Modelo Estadual de Regionalização da Assistência à Saúde. Na atenção às urgências/emergências há predomínio à assistência aos casos clínicos/cirúrgicos de baixa complexidade, como o atendimento aos ferimentos leves e cirurgias de apendicectomias. A atenção à gestante se limita aos partos sem complicações na maioria dos hospitais, havendo necessidade de contratação de recursos humanos (como cardiologistas e neurologistas) para a adequação da oferta. Quanto aos leitos, o maior coeficiente de leitos hospitalares foi de 0,6 leitos/1.000 habitantes no Hospital Regional de Salgueiro, observando-se ainda a concentração de leitos de UTI em apenas três unidades estudadas.

Conclusões: as limitações na estrutura e nos processos de trabalho comprometeram a oferta, a função dos hospitais regionais e consequentemente a assistência hospitalar nas regionais de saúde do Estado.

Palavras-chave Avaliação em saúde, Regionalização, Assistência hospitalar 


\section{Introdução}

A integralidade da atenção à saúde remete, entre outras estratégias, à plena implantação dos três níveis de complexidade assistencial, que poderá ser garantida por meio da conformação e inserção de redes assistenciais nos sistemas de saúde. A concretização dessa imagem-objetivo pressupõe o reconhecimento da interdependência entre os atores e organizações, em face à constatação de que nenhum desses isoladamente terá capacidade suficiente para a solução dos problemas de saúde de uma população em seus diversos ciclos de vida. ${ }^{1}$ Operacionalmente, a rede assistencial deverá prever a integração de uma rede de serviços, com garantia de mecanismos de cooperação e coordenação próprios para uma gestão eficiente e responsável pelos recursos coletivos, a qual responda às necessidades individuais e coletivas. 1,2 Nesse sentido, eliminando-se as barreiras de acesso entre os níveis de atenção, conforma-se o que Hartz e Contandrioupoulos 1 definiram como "sistema sem muros", que se caracteriza pela concretização de sistemas de saúde em nível intermunicipal com vistas à otimização de recursos e ganhos em efetividade para o sistema de saúde.

De acordo com esse pressuposto, o espaço regional torna-se primordial para a organização, gestão e assistência de média e alta complexidade assistencial. A consolidação desse ambiente político-administrativo, no âmbito do Sistema Único de Saúde (SUS), subsidia a construção de uma base sólida para garantia ainda dos princípios de universalidade e equidade, além da descentralização na vertente da regionalização. Almeja-se, nesse sentido, a adequação da oferta de aparatos e tecnologias de saúde de acordo com as necessidades existentes nos diferentes contextos sociais, econômicos e epidemiológicos das regiões e estados do país, que resulta na melhoria dos indicadores de saúde em consequência do melhor acesso da população excluída do sistema público de saúde. ${ }^{3}$ Embora a oferta de serviços de saúde não seja considerada como um dos principais determinantes da melhoria de saúde, a disposição adequada desses serviços e a garantia do acesso universal da população aos equipamentos de saúde em qualidade e cobertura se configuram como um relevante indicador de bem-estar social. 4

Assim, no sistema de saúde regionalizado, a disponibilidade de equipamentos sociais mais próximos aos usuários, amplia a racionalidade dos serviços de saúde de maior densidade tecnológica como a assistência hospitalar. 5 Dessa forma, considerando a complexidade das tecnologias utilizadas e a necessidade de recursos humanos especializados, as unidades hospitalares estarão organizadas para prestar assistência às principais causas de morbimortalidade, direcionando ainda os fundos de saúde municipais, estaduais e federais no sentido das necessidades de saúde da população adscrita, uma vez que esse nível assistencial representa importante parcela orçamentária do SUS.

Por outro lado, apesar dos argumentos favoráveis à implantação de sistemas descentralizados, predomina o modelo assistencial caracterizado pela centralização de hospitais de maior complexidade nas regiões mais urbanizadas e desenvolvidas. Essa desigualdade é observada mesmo nas capitais, onde a periferia é carente de serviços de atenção hospitalar. ${ }^{6}$ Embora seja anseio de gestores e da própria população a disposição de hospitais nos municípios, a oferta organizada e pactuada com a efetiva participação de Secretarias Municipais de Saúde e a ação indutiva das respectivas Secretarias Estaduais terão função preponderante nas ações de programação e regulação desse nível de atenção. ${ }^{7}$

Configurada a oferta organizada, o arranjo de unidades de referência regional, subsidiará o acesso aos hospitais especializados para a população de pequenos municípios, conforme garantido constitucionalmente e normatizado na Norma Operacional de Assistência à Saúde (NOAS/SUS-01/02), 8 mediante as propostas de conformação de micro/macrorregiões, municípios sede de módulo e pólo assistenciais; bem como o apoio à estruturação de consórcios intermunicipais.

Em Pernambuco, o Modelo Estadual de Regionalização da Assistência à Saúde, descrito no Plano Diretor de Regionalização de Pernambuco (PDRPE), ${ }^{9}$ pressupõe a oferta dos equipamentos de saúde de modo equânime em todo o seu território, que está dividido em onze Gerências Regionais de Saúde (GERES). De acordo com esse modelo, o hospital regional corresponde à unidade pública de maior complexidade assistencial, estando dispostos nas GERES localizadas no interior do Estado, cuja função é a garantia do atendimento adequado e de qualidade aos problemas de saúde mais frequentes da população adscrita. Os hospitais regionais são responsáveis pelos seguintes serviços especializados: assistência às situações de urgência e emergência de maior gravidade e aos grupos de risco (gestante de alto risco); bem como pela garantia do acesso da população aos serviços de apoio diagnóstico/terapêutico e às ações especializadas relevantes, como serviços de radiologia, ultrassonografia obstétrica, fisioterapia, cirurgias ambulatoriais e ginecológicas. Além disso, o modelo prevê a existência de leitos para doenças crônicas e a regulação dos leitos 
contratados da rede conveniada ao SUS na sua área de abrangência. 10

Nesse contexto, o relevante papel dos hospitais regionais para implantação do Modelo Estadual de Regionalização da Assistência à Saúde 9 e, portanto, para garantia da assistência integral nas diversas realidades regionais de Pernambuco, foi decisivo para o desenvolvimento da pesquisa apresentada nesse artigo, que tem como objetivo caracterizar a oferta de serviços especializados dos hospitais regionais, considerando os possíveis fatores limitantes para a efetivação deste processo.

\section{Métodos}

Foi feito uma avaliação normativa, 11 mediante a realização de um estudo descritivo no universo dos Hospitais Regionais de Pernambuco, que estão localizados nas regionais de saúde (GERES) do Estado. Cada GERES é constituída por um município sede e um conjunto de municípios circunscritos, conformando um módulo assistencial, de forma que a população dos 184 municípios pernambucanos e do Distrito Estadual de Fernando de Noronha, estimada em 8.413.593 habitantes para o ano 2005,12 tenha assistência à saúde garantida em todas as esferas de atenção. A assistência hospitalar é contemplada por dez unidades dispostas nos municípios de Limoeiro e Palmares (Zona da Mata), Caruaru e Garanhuns (Agreste) e Arcoverde, Salgueiro, Serra Talhada, Afogados da Ingazeira, Ouricuri e Petrolina (Sertão), que devem respeitar o Modelo de Regionalização da Assistência à Saúde do Estado. ${ }^{8}$ Destaca-se que as unidades de Caruaru e Petrolina, além de referência para a média complexidade nas respectivas GERES, apresentam-se ainda como referência macrorregional para todos os níveis de complexidade (Figura 1).

As recomendações do Modelo Estadual de Regionalização, ${ }^{9}$ quanto aos serviços especializados dos hospitais regionais, foram referência para a seleção dos seguintes critérios de avaliação utilizados nessa pesquisa: a) Assistência à urgência e emergência (clínica, pediátrica, traumática e cirúrgica); b) Assistência à gestante de alto risco (prénatal, ultrassonografia e parto de alto risco); c) Oferta de laboratório de análise clínica (próprio ou conveniado à rede privada) e de exames radiográficos (radiografia simples, radiografia com contraste, tomografia computadorizada, ressonância magnética); d) Realização de cirurgias eletivas; e) Disponibilidade de recursos humanos (médicos especialistas disponíveis, em número suficiente e em tempo integral).

O critério de avaliação "assistência à gestante de alto risco" não foi considerado para a avaliação do Hospital Regional de Caruaru (IV Geres). Apesar de integrar o conjunto dos hospitais regionais, essa unidade não presta assistência à gestante, não sendo referência regional para essa especialidade. Para suprir essa necessidade, o município possui dois hospitais públicos, um sob gestão municipal e outro sob dupla gestão (municipal e estadual), com capacidade instalada para assistência ao pré-natal, parto e puerpério, sendo as unidades de referência para esse serviço.

Os demais critérios representam os serviços essenciais à assistência hospitalar de referência regional, devendo ser obrigatoriamente ofertados nas

Figura 1

Localização dos Hospitais Regionais em Pernambuco, de acordo com a Gerência Regional de Saúde (GERES).

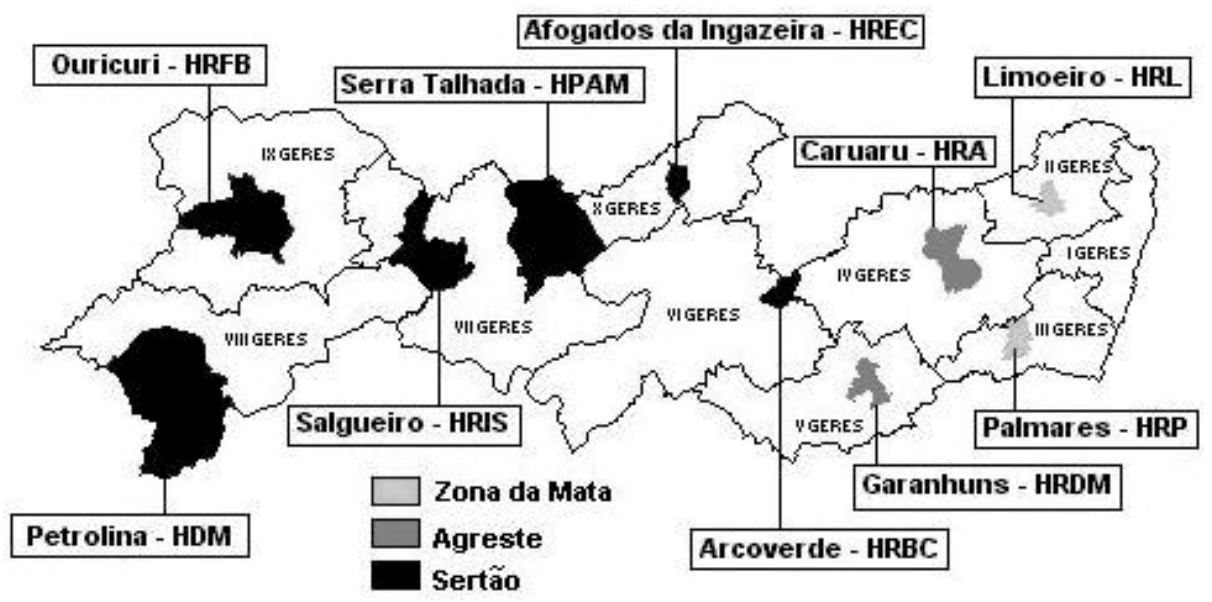


unidades avaliadas. Nesse sentido, a oferta dos referidos serviços será condição para que a assistência seja considerada adequada no presente estudo.

Para a coleta de dados foram realizadas entrevistas estruturadas com diretores (geral, administrativo e clínico) e médicos das referidas unidades, entre novembro e dezembro de 2005 . As questões do instrumento demandaram respostas dicotômicas (do tipo sim ou não), com exceção da assistência aos casos de urgência/emergência. Neste critério, foi elaborada uma pergunta aberta, questionando os procedimentos "mais" e "menos" complexos ofertados no referido setor, no intuito de aferir o nível de complexidade da assistência prestada nos hospitais. A partir desse instrumento, buscou-se ainda identificar as fragilidades da oferta de serviços especializados, considerando os critérios de avaliação como aspectos condicionantes à oferta de serviços, como os recursos humanos, os materiais e equipamentos e a unidade de terapia intensiva (UTI).

Seguindo-se ainda o padrão do Modelo Estadual de Regionalização, 9 identificou-se o quantitativo de leitos (pré-parto, recuperação pós-cirúrgica, cuidados prolongados, UTI), utilizando os dados do Cadastro Nacional de Estabelecimentos de Saúde (CNES), referentes ao ano 2005 (DATASUS, 2007).

O plano de análise dos dados contemplou a caracterização dos serviços especializados, bem como das principais fragilidades para oferta desses serviços, mediante a análise descritiva por critério de avaliação utilizado nesse estudo. Para os leitos hospitalares e de UTI calculou-se a distribuição profissional e o coeficiente de leitos, sendo utilizado para o coeficiente o ano 2004 e a população relativa às GERES correspondentes às unidades hospitalares estudadas (II a X GERES), conformando o número de leitos por 100 mil habitantes de cada regional.

Este estudo foi aprovado pelo Comitê de Ética do Centro de Pesquisas Aggeu Magalhães (CPqAM/FIOCRUZ). Os entrevistados foram convidados a contribuir com a pesquisa, sendo informados sobre os seus objetivos e solicitados a assinarem o Termo de Consentimento Livre e Esclarecido.

\section{Resultados}

Esta avaliação demonstrou que a oferta de serviços especializados nos hospitais regionais se distancia da adequação para assistência hospitalar, conforme preconizado pelo Modelo Estadual de Regionalização da Assistência à Saúde ${ }^{9}$ adotado no presente estudo. Nesse sentido, apresenta-se nessa secção a caracterização da oferta, seguida das principais limi- tações, de acordo com os critérios de avaliação utilizados.

A assistência às urgências e emergências é prestada no universo dos hospitais regionais avaliados. No entanto, considerando a categoria "procedimentos menos complexos", observou-se que a oferta está caracterizada, sobretudo, pelo atendimento aos casos de gripe, de pequenos ferimentos e pela realização de apendicectomias, que foram indicados pelos entrevistados como procedimentos cirúrgicos predominantemente realizados nos hospitais estudados. Entre os "procedimentos mais complexos" ofertados, os entrevistados relataram a assistência aos casos de hipertensão craniana, cardiopatias, hemorragias digestivas, hepatopatias, enfarte agudo do miocárdio grave, acidente vascular cerebral (AVC), assistência ao politraumatizado, entre outros, como indicados na Tabela 1.

A assistência à gestante de alto risco foi caracterizada pela realização acompanhamento pré-natal em apenas uma das unidades avaliadas, bem como de exames de ultrassonografia obstétrica e de partos de baixo e alto risco (normal e cesariana) em todos os hospitais regionais que utilizaram esse item como critério de avaliação. Entretanto, foram identificados alguns fatores condicionantes para a efetivação do parto em gestantes de alto risco, destacando-se a realização dessa ação apenas caso tivesse anestesista de plantão; ou a restrição da realização desse procedimento apenas para as gestantes com eclampsia, cardiopatia e hipertireoidismo, sendo encaminhadas as pacientes acometidas por estenose de válvula mitral ou má formações fetais.

Em relação aos exames complementares, todos os hospitais possuem laboratório de análise clínica, apesar de haver necessidade de material de consumo para o funcionamento adequado desse setor em um dos hospitais avaliados no sertão do Estado. Quanto aos exames de imagem, o conjunto dos hospitais realiza somente radiografia simples. Os procedimentos de maior complexidade, como tomografia computadorizada e ressonância nuclear magnética são realizados, principalmente, em serviços privados localizados nos municípios sede dos hospitais regionais.

A oferta de cirurgias eletivas se resumiu principalmente às cirurgias de pequeno porte (como laparotomia e postectomias) que são realizadas em todos os hospitais avaliados. No entanto, a realização de cirurgias eletivas apresentou-se comprometida pela ausência de cirurgião pediátrico no conjunto das unidades estudadas, pela realização de cirurgias neurológicas apenas nos Hospitais de 
Petrolina e Caruaru, bem como pela limitação de espaço físico (sala de cirurgia e leitos) em quatro hospitais regionais abordados.

Considerando os recursos humanos, todos os gestores entrevistados apontaram que os hospitais possuem médico clínico geral, pediatra, ginecologista/obstetra e anestesista, com exceção da unidade de Caruaru que, por não prestar assistência à gestante, não apresenta ginecologistas/obstetras no seu quadro de profissionais. Observou-se ainda a presença diária de um ou mais clínicos, apesar de ter sido frequentemente identificado no presente estudo que esses especialistas realizam procedimentos além da sua área de atuação. Esse fato ocorreu, sobretudo, em hospitais do sertão, onde alguns clínicos de plantão realizam partos; e outros são concursados como clínicos e atuam como pediatra, caso também tenha essa especialidade. Quanto aos anestesistas, apesar de os dados mostrarem a existência de especialistas contratados no universo dos hospitais avaliados (Tabela 2), entrevistados em quatro unidades abordadas referem à necessidade de recorrer às cooperativas para que esse profissional esteja presente em alguns plantões.

Da mesma forma, apenas quatro unidades apresentam neurologista no quadro profissional, estando presente em todos os plantões somente nos hospitais de Caruaru e Petrolina. A contratação deste profissional foi apontada como necessária por entrevis- tados de oito hospitais, inclusive naqueles que apresentam o profissional de plantão, embora em quantidade insuficiente para suprir a demanda. O mesmo ocorre em relação aos cardiologistas, que estão ausentes em um dos hospitais avaliados e, em cinco unidades, esses especialistas estão contratados em número insuficiente (Tabela 2).

Seguindo as informações dos gestores entrevistados, observou-se que, com exceção de um hospital localizado no sertão do Estado, o universo dos hospitais apresenta cirurgião geral no quadro de profissionais. Entretanto, a maioria dos hospitais não dispõe desse especialista diariamente, sendo indicada pelos entrevistados a necessidade de contratação de maior quantidade de cirurgiões gerais. Da mesma forma, quatro profissionais entrevistados consideram necessária a vinculação de cirurgiões pediátricos e neurocirurgiões aos plantões, sendo este último especialista contratado apenas nas unidades de Caruaru e Petrolina (Tabela 2).

Ainda em relação à necessidade de contratação de plantonistas permanentes, gestores e médicos entrevistados de hospitais do Sertão apontaram um número significativo de profissionais em regime de sobreaviso (plantão à distância), o que dificulta a evolução e resolução dos casos. Foi também considerado urgente a necessidade de treinamento, reciclagem profissional e implementação de uma política de incentivo financeiro para os profissionais

Tabela 1

Assistência às urgências e emergências clínicas nos Hospitais Regionais de Pernambuco, 2005.

\begin{tabular}{|c|c|c|c|c|}
\hline $\begin{array}{l}\text { Nível de } \\
\text { complexidade }\end{array}$ & Clínica & Pediátrica & Traumática & Cirúrgica \\
\hline $\begin{array}{l}\text { Procedimentos } \\
\text { mais } \\
\text { complexos }\end{array}$ & $\begin{array}{l}\text { Hipertensão craniana; } \\
\text { Cardiopatias; } \\
\text { Hemorragias digestivas; } \\
\text { Hepatopatias; } \\
\text { Enfarte agudo do } \\
\text { miocárdio grave; } \\
\text { Acidente vascular } \\
\text { cerebral (AVC) }\end{array}$ & $\begin{array}{l}\text { Pneumonia; } \\
\text { Septicemia grave; } \\
\text { Cardiopatia } \\
\text { congênita; } \\
\text { Infecções } \\
\text { respiratórias } \\
\text { agudas agravadas } \\
\text { (pneumonias e } \\
\text { derrame pleural) }\end{array}$ & $\begin{array}{l}\text { Politraumatismo;* } \\
\text { Trauma toráxico; } \\
\text { Traumas abdominais } \\
\text { abertos e fechados }\end{array}$ & $\begin{array}{l}\text { Abdômen agudo; } \\
\text { Úlcera perfurada; } \\
\text { Abdômen agudo } \\
\text { pós-infecção biliar; } \\
\text { Laparotomia; } \\
\text { Trauma Crânio- } \\
\text { cefálico (TCE) }\end{array}$ \\
\hline $\begin{array}{l}\text { Procedimentos } \\
\text { menos } \\
\text { complexos }\end{array}$ & \multicolumn{2}{|c|}{$\begin{array}{l}\text { Gripe, hipertermia, desidratação, cefaléia, } \\
\text { diarréia, infecção intestinal, odontalgia, } \\
\text { pequenos ferimentos. }\end{array}$} & $\begin{array}{l}\text { Fraturas fechadas e } \\
\text { agressões leves por } \\
\text { arma de fogo e arma } \\
\text { branca. }\end{array}$ & $\begin{array}{l}\text { Apêndice } \\
\text { supurado }\end{array}$ \\
\hline
\end{tabular}

*Apenas para os Hospitais de Caruaru e Petrolina. 
aderirem aos serviços de saúde localizados no interior de Pernambuco.

$\mathrm{Na}$ análise dos leitos hospitalares, observou-se que oito hospitais apresentam leitos de recuperação pós-cirúrgica e que os leitos específicos para préparto são contemplados em seis unidades regionais. Diferentemente, os leitos hospitalares para cuidados prolongados estão presentes apenas em quatro unidades avaliadas (Tabela 3). Consequentemente, pacientes sequelados de Acidente Vascular Cerebral (AVC), por exemplo, ocupam leitos de UTI, devido à ausência de enfermaria adequada, de fisioterapeuta e auxiliares de enfermagem capacitados. Em um hospital do Agreste, para a internação que necessite de cuidados prolongados, são utilizados leitos específicos, através de um convênio com um hospital próprio do SUS, localizado em município vizinho.

Tabela 2

Distribuição de profissionais contratados por especialidades médicas nos Hospitais Regionais de Pernambuco, 2005.

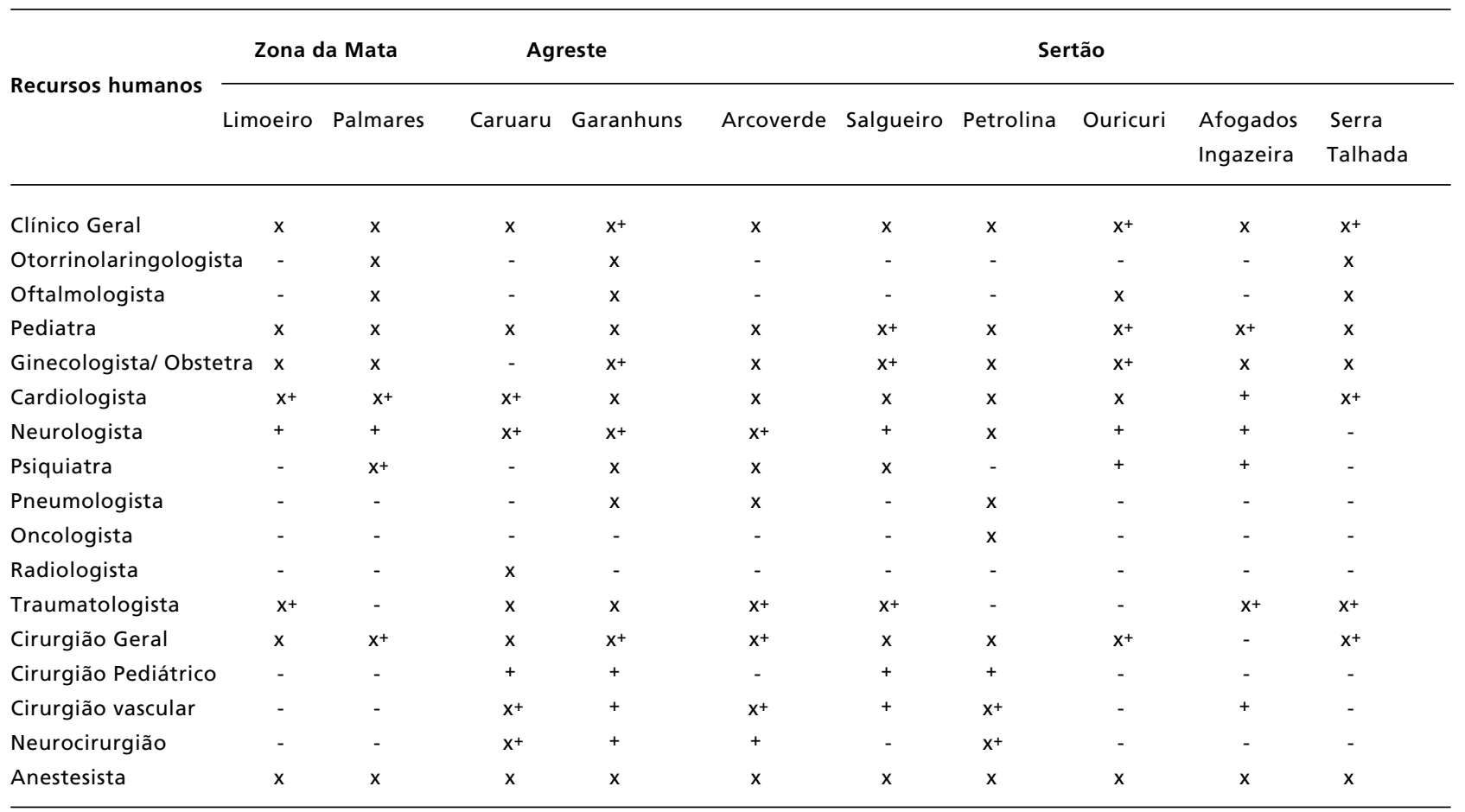

x Especialista contratado em número suficiente; $x+$ Especialista contratado em número insuficiente; +Especialista não contratado, mas com necessidade de contratação; - Especialista não contratado.

Tabela 3

Distribuição de tipos de leitos nos Hospitais Regionais de Pernambuco, 2005.

\begin{tabular}{|c|c|c|c|c|c|c|c|c|c|c|}
\hline \multirow{2}{*}{ Tipos de leitos } & \multicolumn{2}{|c|}{ Zona da Mata } & \multicolumn{2}{|c|}{ Agreste } & \multicolumn{6}{|c|}{ Sertão } \\
\hline & Limoeiro & Palmares & Caruaru & Garanhuns & Arcoverde & Salgueiro & Petrolina & Ouricuri & $\begin{array}{l}\text { Afogados } \\
\text { Ingazeira }\end{array}$ & $\begin{array}{l}\text { Serra } \\
\text { Talhada }\end{array}$ \\
\hline Pré-parto & $x$ & $x$ & - & $x$ & - & $x$ & $x$ & - & $x$ & - \\
\hline Cuidados prolongado & os & - & - & - & - & $x$ & $x$ & - & $x$ & $x$ \\
\hline
\end{tabular}


Em relação ao quantitativo de leitos disponíveis observa-se na Tabela 4 que a maioria se concentra nos hospitais de Caruaru e Petrolina, que abrangem respectivamente $15,3 \%$ e $17,3 \%$. As unidades que possuem o maior coeficiente de leitos por 1.000 habitantes são o Hospital de Salgueiro (0,6/1.000 hab.), seguido do Hospital de Petrolina e de Arcoverde, que apresentaram coeficiente de 0,5 leitos/1.000 hab. Em situação oposta encontra-se o Hospital de Limoeiro, que contempla apenas 5,2\% dos leitos e possui 0,1 leitos/1.000 hab. Os leitos em UTI foram identificados apenas nos hospitais de Caruaru, Petrolina e Arcoverde, que apresentam proporções desse equipamento em relação ao número total de leitos respectivamente de $5,7 \%$, $3,0 \%$ e $3,6 \%$.
A ausência de leitos de UTI foi identificada como fator limitante para a resolutividade de casos clínicos e cirúrgicos, e consequentemente para a oferta de serviços especializados de alta complexidade assistencial. Por outro lado, no Hospital Regional de Ouricuri, que se encontrava em precárias condições de trabalho devido a uma reforma estrutural, um médico entrevistado concordou com a importância da implantação de UTI no referido hospital, mas destacou que tal recurso não é prioridade para uma unidade hospitalar ainda carente de recursos humanos, como anestesista, cirurgião geral e ginecologista/obstetra.

Tabela 4

Distribuição proporcional e coeficiente de leitos hospitalares (por 1.000 hab.), de acordo com os Hospitais Regionais de Pernambuco, 2005.

\begin{tabular}{|c|c|c|c|c|c|c|}
\hline \multirow{2}{*}{ Hospitais } & & \multicolumn{3}{|c|}{ Total de leitos } & \multicolumn{2}{|c|}{ Leitos de UTI* } \\
\hline & & $\mathrm{n}$ & $\%$ & coef & $\mathrm{n}$ & $\%$ \\
\hline \multirow[t]{2}{*}{ Zona da Mata } & Limoeiro & 60 & 5,2 & 0,1 & - & - \\
\hline & Palmares & 105 & 9,1 & 0,2 & - & - \\
\hline \multirow[t]{2}{*}{ Agreste } & Caruaru & 176 & 15,3 & 0,2 & 10 & 5,7 \\
\hline & Garanhuns & 110 & 9,6 & 0,2 & - & - \\
\hline \multirow[t]{6}{*}{ Sertão } & Arcoverde & 166 & 14,5 & 0,5 & 6 & 3,6 \\
\hline & Salgueiro & 123 & 10,7 & 0,6 & - & - \\
\hline & Petrolina & 199 & 17,3 & 0,5 & 6 & 3,0 \\
\hline & Ouricuri & 52 & 4,5 & 0,2 & - & - \\
\hline & Afogados da Ingazeira & 66 & 5,7 & 0,2 & - & - \\
\hline & Serra Talhada & 91 & 7,9 & 0,3 & - & - \\
\hline Total & & 1148 & 100,0 & 0,2 & 22 & 1,9 \\
\hline
\end{tabular}

* Percentual de leitos de UTI em relação ao número total de leitos hospitalares; coef=coeficiente. Fonte: DATASUS/CNES/2005.12

\section{Discussão}

Não obstante ser consensual que a política de regionalização da assistência à saúde seja efetiva desde que seus princípios sejam respeitados, as iniciativas de avaliação da sua real contribuição para fortalecimento do SUS ainda são incipientes. No presente estudo, a complexidade da assistência prestada e a contratação de recursos humanos foram os principais fatores limitantes da oferta nos hospitais de referência regional de Pernambuco. Adotando o Modelo
Estadual de Regionalização da Assistência à Saúde 9 como padrão de referência, pôde-se ainda constatar que a resolutividade dos casos clínicos e cirúrgicos, bem como a oferta de exames complementares para diagnóstico, se apresentaram incompatíveis com o preconizado.

A desorganização da assistência à saúde descortina a persistência de um modelo assistencial hospitalocêntrico, evidenciando a fragmentação dos três níveis assistenciais. Os hospitais avaliados persistem como referência para os casos clínicos de 
baixa complexidade, permitindo o questionamento sobre a cobertura, aceitação e resolutividade das unidades de atenção primária nos municípios do Estado. Ademais, esse modelo agrega como consequência o desperdício e o gasto erroneamente direcionado de recursos, comprometendo a qualidade assistencial em âmbito hospitalar.7 O perfil da oferta concentrada na assistência primária em hospitais de referência evidencia um sistema de saúde fragmentado com efeitos distantes da imagem-objetivo almejada. Efeitos esses provavelmente ainda consagrados nos sistemas estaduais, como o pernambucano, que apresentam: foco na doença ou na prevenção individual, baixa responsabilização pela saúde de uma população definida; descontinuidade no atendimento; e precariedade, sobretudo dos serviços de maior densidade tecnológica, com indução da demanda pela oferta de serviços. ${ }^{2}$

As instalações de leitos, equipamentos, bem como a contratação de médicos especializados, protagonizaram entre as fragilidades da oferta de serviços nos hospitais avaliados. Esse cenário corrobora com o apresentado por Ferla et al.,13 ao analisarem os hospitais regionais do Rio Grande do Sul, e com o estudo de Carvalho et al., 14 que, a partir da perspectiva do gestor municipal, demonstraram como relevante deficiência a carência de profissionais nos hospitais regionais de Pernambuco.

No presente estudo, a distribuição e fixação quantitativa/qualitativa dos profissionais, principalmente de cirurgiões e neurologistas, foram categóricos para a complexidade dos serviços ofertados, figurando ainda entre os seus principais fatores limitantes. Em relação a esse aspecto, destacam-se as unidades do sertão, que estão distantes dos centros mais desenvolvidos, em municípios com aparelhos e equipamentos urbanos ainda incipientes e com dificuldades de acesso pelas rodovias estaduais e federais, fatores que podem influenciar acentuadamente na contratação de médicos especializados. Nesse sentido, a instalação de mecanismos compensatórios para atrair profissionais especializados a essas localidades, e mais especificamente para as unidades hospitalares de caráter regional, poderia contribuir para a redução das iniquidades presentes na oferta e consequentemente na utilização dos serviços de saúde.

Em relação aos leitos, o Brasil apresenta grande inadequação na oferta, seja pelo excesso em algumas regiões, ausência em outras ou por deficiências em qualidade. 15 Ademais, é crescente o surgimento de pequenos hospitais, com média de leitos inferior a 30, que apresentam baixa resolutividade e com criação possivelmente mais vinculada a interesses políticos do que a interesses técnicos. ${ }^{16}$ No presente estudo, o número de leitos se concentrou em condição intermediária entre os "pequenos hospitais" e a escala mínima de 200 leitos referida pela literatura internacional. ${ }^{17} \mathrm{O}$ coeficiente de leitos para a população adscrita pelos hospitais regionais demonstrou ainda grave defasagem em relação aos parâmetros preconizados (03 a 04 leitos/1.000 hab.) pela Portaria GM 1.101/02.18

A despeito desse cenário, os entrevistados não apontaram a insuficiência de leitos como um problema relevante, uma vez que a referência dos pacientes para garantia da resolutividade apresentase mais diretamente relacionada às limitações da capacidade assistencial do hospital. Nesse sentido, as unidades avaliadas aproximam-se da situação apresentada por Mendes 17 sobre os hospitais de pequeno porte, que devido às limitações na complexidade assistencial internam o suficiente para garantir a referência para hospitais regionais. Entretanto, cabe ressaltar que os hospitais avaliados são de médio e grande porte, se apresentando como referência regional, no modelo assistencial previsto no Estado.

Diferentemente, a ausência de leitos em UTI incluiu-se entre os determinantes para a resolutividade de casos que exigem tal aparato tecnológico. Verificou-se que, com exceção da unidade regional de Arcoverde, os demais hospitais que ofertam esse serviço estão localizados nas unidades de Caruaru e Petrolina, que são referência macrorregional no Modelo Estadual de Regionalização. 9 Entretanto, considerando que a Portaria GM 1.101/0218 indica a necessidade de $4 \%$ a $10 \%$ de leitos de UTI em relação ao número total de leitos, apenas o Hospital Regional de Caruaru se enquadrou nesse padrão.

Apesar das fragilidades observadas na oferta de serviços especializados nos hospitais avaliados em Pernambuco, tais características acompanham o processo em curso para a implantação da rede hospitalar no sistema de saúde brasileiro. Os desafios assumidos pelo SUS para a consolidação do modelo assistencial, universal, integral, regionalizado e hierarquizado, apresentaram forte influência da municipalização do setor saúde, e as iniciativas vinculadas à atenção básica apresentaram maiores avanços do que a gestão dos serviços hospitalares, sobretudo a partir de 1994 com a implantação da Estratégia de Saúde da Família. 19 No período 1997-2001 verificou-se um crescimento médio de $102 \%$ nos investimentos do Ministério da Saúde, mormente relativos ao custeamento de internações e procedimentos de 
média e alta complexidade. Por outro lado, no ano de 2001, o número de leitos apresentou redução média de 3,2\%, com maior impacto para os leitos privativos lucrativos que sofreram significativa redução de $27 \% .^{20}$

Nesse contexto, o papel dos organismos internacionais, no sentido de garantir a sustentabilidade das políticas sociais mediante ao incentivo, apoio e avaliação das iniciativas de extensão do acesso aos serviços de qualidade, 21 influenciam o percurso da política de investimentos do SUS. Estratégias como o Projeto de Reforço à Reorganização do SUS (REFORSUS), 22 desde 1996, e o Projeto de Investimentos para a Qualificação do Sistema Único de Saúde (QUALISUS), 23 instituído em 2004, ganharam destaque. Enquanto a primeira, em acordo com o Banco Mundial (BIRD), Banco Interamericano de Desenvolvimento (BID) e as três esferas de governo investiram 750 milhões de dólares para a reforma da rede hospitalar, aquisição de equipamentos e desenvolvimento de sistemas de gerenciamento; 22 a segunda iniciou em 27 regiões metropolitanas (cerca de 80 hospitais com serviços de emergência), visando o acolhimento ao paciente, além de uma atenção à saúde mais efetiva, com prioridade aos casos mais graves e com redução do tempo de permanência hospitalar. 23

Conforme o Relatório Final do REFORSUS em Pernambuco, $60 \%$ dos hospitais regionais receberam investimentos referentes a esse projeto. Entretanto, com exceção da unidade de Arcoverde, nos demais hospitais os equipamentos e reformas adquiridos tiveram como principal objetivo a melhoria da atenção à saúde materno-infantil.24 Observou-se, na presente avaliação, que embora a atenção à gestante de alto risco seja reconhecidamente prioritária em hospitais de referência regional, essa não é realizada adequadamente na maioria das unidades abordadas. Apesar de o investimento ter sido direcionado para a atenção materno-infantil, o comprometimento dessa área sugere a persistência de limitações de contratação e de profissionais qualificados; bem como da estruturação com equipamentos e materiais direcionados à referida prática.

Os percalços observados na assistência aos casos de urgência/emergência e nos procedimentos cirúr- gicos nos hospitais regionais põem em julgamento as prioridades de alocação de recursos do REFORSUS no Estado. No presente estudo revelouse uma lacuna entre a oferta de serviços, a manutenção da capacidade instalada e a realização de investimentos públicos, sobretudo aqueles advindos de empréstimos internacionais, como o REFORSUS, que incrementam a dívida pública do país e consequentemente contribuem para a redução do investimento nacional para a prestação de tais serviços. ${ }^{20}$

Direcionada aos serviços de urgência/ emergência, a Política QUALISUS foi avaliada em três hospitais da região metropolitana do Recife, demonstrando grau de implantação incipiente. Como principais obstáculos à implantação, foram apontados: a deficiência de profissionais especializados, a baixa resolutividade das demais unidades de atenção às urgências/emergências e o atraso da efetivação pelo Ministério da Saúde das melhorias estruturais previstas na política. 25 Mesmo referindo-se à hospitais localizados em Recife, que é eixo central da rede assistencial do Estado, sendo referência (inter)estadual, as lacunas observadas na avaliação do QUALISUS demonstram estreita relação com as principais limitações ressaltadas nos hospitais regionais avaliados no presente estudo, quer seja a carência de profissionais especializados, a resolutividade ou as condições estruturais necessárias à efetivação de um atendimento digno.

Nesse sentido, a configuração da real missão dos hospitais regionais e sua inserção no Modelo Estadual de Regionalização da Assistência à Saúde9 será indispensável para a redefinição das políticas específicas para cada realidade loco-regional. Tal passo subsidiará a conformação de um sistema de saúde com integralidade e flexível o suficiente para respeitar as divergências existentes no perfil geográfico, no desenvolvimento urbano e regional, no desenvolvimento econômico e social, na identidade cultural local, no perfil epidemiológico, nas condições sanitárias, bem como na capacidade instalada de serviços de saúde já existentes em cada uma das regionais de saúde do Estado de Pernambuco.

\section{Referências}

1. Hartz ZMA, Contandriopoulos A-P. Integralidade da atenção e integração de serviços de saúde: desafios para avaliar a implantação de um "sistema sem muros". Cad Saúde Pública. 2004; 20 (suppl. 2): S331-6.
2. Costa-e-Silva V, Rivera FJU, Hortale VA. Projeto Integrar: avaliação da implantação de serviços integrados de saúde no Município de Vitória, Espírito Santo, Brasil. Cad Saúde Pública. 2007; 23: 1405-14. 
3. Arretche M. Mitos da descentralização: mais democracia e eficiência nas políticas públicas? Rev Bras Ciênc Soc. 1996; 31: 44-66.

4. Borrell C. Desigualdades y servicios de salud. Saúde Soc. 2006; 15: 9-22.

5. Mato Grosso. Secretaria de Saúde. Júlio Strubing Muller Neto (org.). A regionalização da saúde em Mato Grosso: em busca da integralidade da atenção. Cuiabá, MT; 2002. 336p.

6. Castelar RM; Mordelet P, Grabois V. Gestão hospitalar - um desafio para o hospital brasileiro. Cooperação BrasilFrança: Édition École Nationale de la Santé Publique; 1995.

7. Vecina Neto G, Malik AM. Tendências na assistência hospitalar. Ciênc Saúde Coletiva. 2007, 12: 825-39. Disponível em: http://www.scielo.br/scielo [2007 Jul 04].

8. Brasil. Norma Operacional de Assistência à Saúde NOAS/SUS - 01/2001. Portaria no 95, de 26 de janeiro de 2001. Brasília; 2001b. 110 p.

9. Pernambuco. Secretaria Estadual de Saúde. Plano Diretor de Regionalização de Pernambuco; 2001. 48 p.

10. Pernambuco. Secretaria Estadual de Saúde. A Gestão: responsabilidades; 1998.

11. Contrandriopoulos A-P, Champagne F, Denis J, Pineault R. A avaliação na área de saúde: conceitos e métodos. In: Hartz ZMA, organizadora. Avaliação em saúde: dos modelos conceituais à prática na análise da implantação de programas. Rio de Janeiro: Fiocruz; 1997. p. 29-47.

12. Datasus. Cadastro Nacional de Estabelecimentos de Saúde (CNES), 2007. [acesso em: 13 jun 2007]. Disponível em: www.datasus.gov.br/cnes

13. Ferla AA, Geyer LMC, Pritoluk LB, Breunig M. A regionalização da atenção à saúde na experiência da gestão estadual do SUS no Rio Grande do Sul. Porto Alegre: Secretaria de Saúde do Rio Grande do Sul; 2002.

14. Carvalho EF, Cesse EAP, Machado, HOP. Fatores Limitantes e facilitadores de mudança nas organizações de saúde do SUS. In: Freese E. (org.) Municípios: a gestão da mudança em saúde. Recife: Ed. Universitária da UFPE; 2004. p. 233-59.

15. Castro MSM, Travassos C., Carvalho MS. Efeito da oferta de serviços de saúde no uso de internações hospitalares no Brasil. Rev. Saúde Pública. 2005; 39,2: 277-284.

Recebido em 18 de fevereiro de 2008 Versão final apresentada em 17 de agosto de 2009

Aprovado em 18 de setembro de 2009
16. Santos MAB, Gerschman S. As segmentações da oferta de serviços de saúde no Brasil - arranjos institucionais, credores, pagadores e provedores. Ciênc Saúde Coletiva. 2004; 9: 795-806.

17. Mendes EV. Reflexão sobre a NOAS SUS 01/02. In: Relatório final do $1^{\circ}$ Seminário do CONASS para a construção de Consensos: preocupações e prioridades dos Secretários Estaduais de Saúde quanto à organização, gestão e financiamento do SUS/Conselho Nacional de Secretários de Saúde. Brasília: CONASS; 2003. p. 65-100.

18. Brasil. Ministério da Saúde. Portaria GM n. 1101, de 12 de junho de 2002. Estabelece parâmetros assistenciais do SUS. Diário Oficial da União [DOU], v.139, (112): 36, 13 jun. 2002. Seção 1. Brasília, DF.

19. Bahia L. O mercado de planos de saúde no país: tendências pós-regulamentação. In: Negri DG (org.). Brasil: radiografia da saúde. Campinas: Instituto de Economia, Unicamp; 2001. 345 p.

20. Soares A. Formação e desafios do sistema de saúde no Brasil: uma análise de investimentos realizados para ampliação da oferta de serviços. Cad Saúde Pública. 2007; 23 : 1565-72.

21. Bermudez J. Acesso a insumos em saúde: desafios para o Terceiro Mundo. Cad Saúde Pública. 2007; 23: 744-5.

22. Brasil. Ministério da Saúde. Secretaria de Assistência à Saúde. Reforsus - prioridades de investimentos. Brasília, DF; 1996. (documento interno).

23. Brasil. Ministério da Saúde. Secretaria de Assistência à Saúde. Qualisus. Brasília, DF; 2007. [acesso em: 13 jun. 2007]. Disponível em: http://portal.saude.gov.br/SAUDE/ area.cfm?id_area $\mathbf{4 0 8}$

24. Pernambuco. Secretaria Estadual de Saúde. Projeto REFOSUS em Pernambuco - Relatório Final; 2005. 78p.

25. Gusmão-Filho FAR. Análise de implantação da Política de Qualificação da Atenção à Saúde do Sistema Único de Saúde - Política Qualisus - em três hospitais do município do Recife. [tese]. Recife: Centro de Pesquisas Aggeu Magalhães da Fundação Oswaldo Cruz. 2008. 181 p. 